\title{
Subnanometer Local Temperature Probing and Remotely Controlled Drug Release Based on Azo-Functionalized Iron Oxide Nanoparticles
}

\author{
Andreas Riedinger, ${ }^{\dagger}$ Pablo Guardia, ${ }^{\dagger}$ Alberto Curcio, ${ }^{\dagger}$ Miguel A. Garcia, ${ }^{\dagger}$ Roberto Cingolani, ${ }^{\dagger}$ \\ Liberato Manna, ${ }^{\dagger}$ and Teresa Pellegrino* ${ }^{*},, \S$ \\ $\dagger$ Istituto Italiano di Tecnologia, Via Morego 30, 16163 Genova, Italy \\ "Instituto de Ceramica y Vidrio, CSIC \& IMDEA Nanociencia, E-28049, Madrid, Spain \\ ${ }^{\S}$ National Nanotechnology Laboratory of CNR-NANO, via per Arnesano km 5, 73100 Lecce, Italy
}

Supporting Information

ABSTRACT: Local heating can be produced by iron oxide nanoparticles (IONPs) when exposed to an alternating magnetic field (AMF). To measure the temperature profile at the nanoparticle surface with a subnanometer resolution, here we present a molecular temperature probe based on the thermal decomposition of a thermo-sensitive molecule, namely, azobis[N-(2-carboxyethyl)-2-methylpropionamidine]. Fluoresceineamine (FA) was bound to the azo molecule at the IONP surface functionalized with poly(ethylene glycol) (PEG)

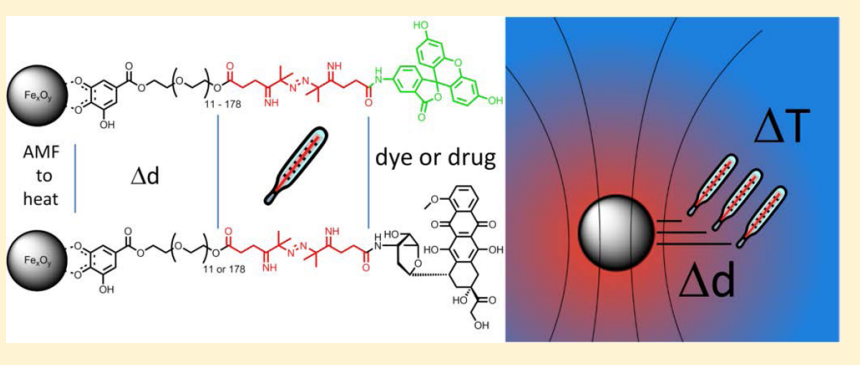
spacers of different molecular weights. Significant local heating, with a temperature increase up to $45^{\circ} \mathrm{C}$, was found at distances below $0.5 \mathrm{~nm}$ from the surface of the nanoparticle, which decays exponentially with increasing distance. Furthermore, the temperature increase was found to scale linearly with the applied field at all distances. We implemented these findings in an AMF-triggered drug release system in which doxorubicin was covalently linked at different distances from the IONP surface bearing the same thermo-labile azo molecule. We demonstrated the AMF triggered distance-dependent release of the drug in a cytotoxicity assay on KB cancer cells.

KEYWORDS: Fluorescent thermometer, temperature probe, magnetic nanoparticles, temperature profile, drug release, azo-compounds

$\mathrm{T}$ emperature measurements with high spatial resolution and accuracy at the nanoscale are an active area of research, especially since inorganic nanoparticles were demonstrated to act as heat foci under different excitation sources (i.e., alternating magnetic field (AMF) or radiofrequency field). Magnetic nanoparticles (MNPs), indeed, under an AMF exposure can be used as heat mediators to treat tumor tissues in the so-called magnetic induced hyperthermia. ${ }^{1-8}$ Besides, MNPs have been also proposed as drug carriers with remote release actuation triggered by the heat generated upon exposure to an $\mathrm{AMF} .^{9-11}$

It is generally assumed that significant heating occurs only in the very close vicinity of the MNP surface. ${ }^{12}$ Several works have now accumulated indirect proofs that, even if no macroscopic temperature changes under AMF are recorded, the local temperature at the nanoparticle surface might be significantly different from that of the surroundings. It was shown for instance that, even if the concentration of the MNPs at the tumor cells did not produce macroscopic heating, MNPs could induce the apoptosis of tumor cells under AMF. ${ }^{13}$ Pralle and co-workers monitored the local temperature increase of the MNPs at the cell surface, by looking at the thermally activated TRPV1 ion channels, as these protein channels allow the influx of calcium ions whenever local temperature reaches about
$40{ }^{\circ} \mathrm{C} .{ }^{14}$ Even in the work of Pralle et al. no macroscopic temperature increase was recorded during the measurements. ${ }^{14}$

To date, it is still challenging to quantify the local temperature profile of MNPs excited via an AMF. Classical methods for heat characterization (e.g., specific absorption rate (SAR) measurements) are not suitable to measure local heating effects, for the following reasons: (i) the experiments have to be performed with highly concentrated dispersions of MNPs, where macroscopic heating of the environment occurs and interparticle interactions cannot be excluded; (ii) the SAR definition is only valid when the experiments are performed in nearly adiabatic conditions, which are far from the conditions for a diluted solution where heat losses are dominant and hence the system is isothermal; and (iii) the temperature recorded corresponds to the macroscopic temperature in the medium in which the MNPs are dispersed rather than the temperature at the nanoparticle surface.

Some attempts toward temperature mapping strategies at the nanoscale to measure the temperature at the nanoparticle surface were recently reported, however without addressing the temperature gradient issue. ${ }^{15,16}$ Jacobson et al. reported a

Received: January 15, 2013

Revised: April 9, 2013 

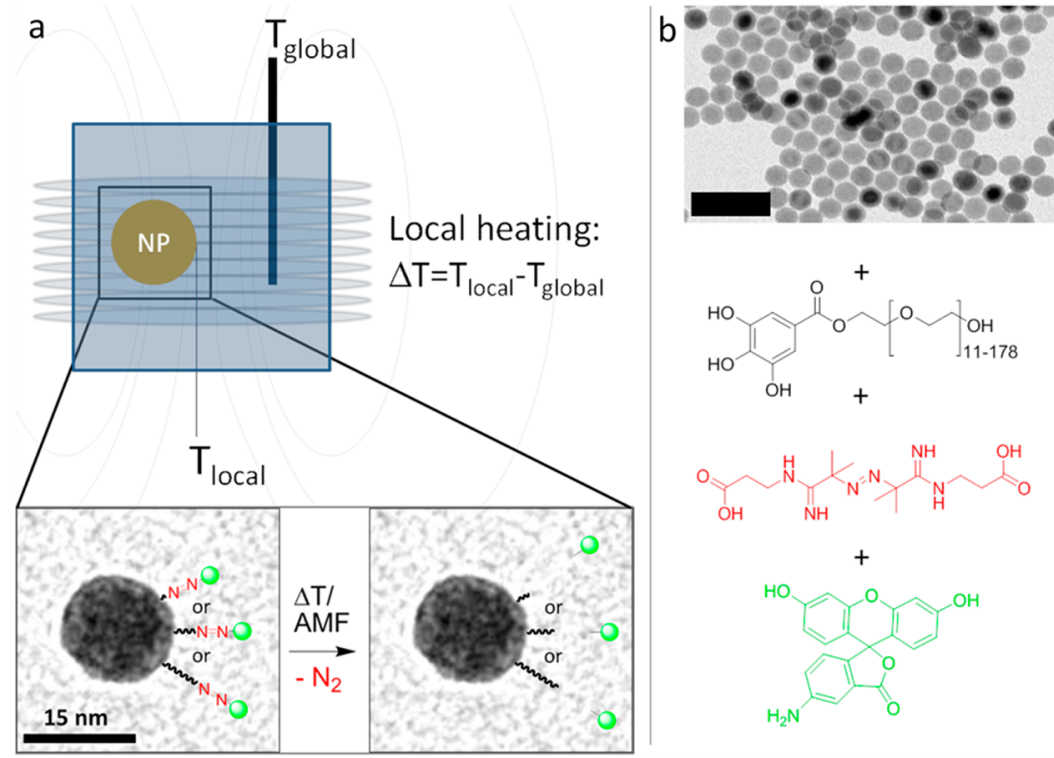

Figure 1. Principle of $\Delta T$ measurements and used building blocks. (a) Sketch of the local-to-global temperature differences measurement principle in a hyperthermia device. A fluorescence dye linked to the IONPs through azo-PEG spacer groups decomposes as a function of temperature and time, and the dye release enables local temperature measurements. Global temperatures are assessed by an optical temperature probe. (b) Building blocks of the local temperature probe. The $15 \mathrm{~nm}$ spherical IONPs (scalebar $50 \mathrm{~nm}$ ) are functionalized stepwise with gallol-PEG $\left(M_{\mathrm{w}}=500,1500\right.$, or $8000 \mathrm{Da})$, an azo-molecule (VA057), and fluoresceine amine (FA).

temperature molecular probe based on the dehybridization of double strand DNAs bound to gold nanoparticles occurring under the application of a radio frequency field. ${ }^{17}$ The DNA melting was attributed to the local heating of gold nanoparticles induced by eddy currents. ${ }^{18}$ Kotov et al. have developed a nanothermometer based on the molecular spring assemblies ${ }^{19}$ which could be applied to measure the gold nanoparticle temperature. In their structure, the gold particles and the CdTe particles were connected via a polymeric spacer that acted as a molecular spring. Since a temperature variation from 20 to 50 ${ }^{\circ} \mathrm{C}$ results in an expansion of the polymer, the interaction between exciton-plasmon pairs could be varied, thus resulting in a variation of the fluorescence signal. This system, despite of its high spatial resolution, shows some drawbacks due to the intrinsic working principle used; (i) the method can be only applied to plasmonic nanoparticles, and (ii) the distance between the semiconductor and the plasmonic particle should ensure changes in the fluorescence enhancement. Absolute temperature measurements at the nanoscale were recently demonstrated by Carlos et al. using a luminescent molecular thermometer based on rare hearth chelating agents, which were embedded into the silica shell at the surface of maghemite nanoparticles. ${ }^{20}$ The temperature dependence of the emission line of the $\mathrm{Tb}^{3+}$ dopant with respect to another emission line of the $\mathrm{Eu}^{3+}$ dopant, which remains constant over the entire temperature range, allows for the absolute temperature measurement. It has also been demonstrated that this system has an accuracy of $0.5{ }^{\circ} \mathrm{C}$ in a wide temperature interval, including that of the physiological conditions. ${ }^{20}$ Moreover, because this system contains magnetic nanoparticles it has been proposed as an in situ thermometer for the real time temperature sensing during magnetic hyperthermia. So far however it has not been exploited for such purpose.

In this regard, Rinaldi and Polo-Corrales ${ }^{21}$ have developed an iron oxide thermo-responsive fluorescent polymer system (poly $(N$-isopropylacrylamide-fluorescent modified acrylamide), $\mathrm{p}$ (NIPAM-co-FMA)) in which the fluorescence intensity change of benzofuran type fluorophores attached to the polymer is correlated to the temperature at the iron oxide nanoparticle surface. Under an AMF, the fluorescence intensity of the dye indicates a local temperature above $35{ }^{\circ} \mathrm{C}$ (which corresponds to the phase transition temperature of the polymer attached at the nanoparticle surface), even if the medium temperature was stable at $20{ }^{\circ} \mathrm{C}$. Once the AFM was switched off, the nanoparticle temperature surface reached thermal equilibrium with the surroundings. In this case, only changes above the lower critical solution temperature, LCST, that is, 35 ${ }^{\circ} \mathrm{C}$, could be monitored, but no temperature profile at the MNP surface could be measured.

To date, all methods developed for local temperature measurements at the nanoscale are either nanoparticle type specific or lack in spatial information. Hence, no mapping of the temperature gradients at the surface of MNPs exposed to AMF has been reported so far. This information indeed plays a key role for the prediction of heat effect of magnetic nanoparticles induced by AMF and for the design of new therapeutic probes. Here, to measure the local temperature changes with spatial resolution at subnanometer scale $(0.5 \mathrm{~nm})$ on IONPs when exposed to an AMF, we have designed a fluorescent probe thermometer system which exhibits the following characteristics: (i) it works with highly diluted solutions (5 $\mathrm{nM}$ particles concentration), where interparticle interactions are minimized and isothermal conditions are ensured; (ii) it measures at distinct distances from the nanoparticle surface in order to map the temperature gradient by linking a dye-thermo-labile group through different macromolecular spacers (PEG) to the IONPs, and (iii) it measures over a long time lapse $(1 \mathrm{~h})$, which allows evaluating the difference between global and local temperature in steadystate conditions.

In our approach, the fluoresceineamine (FA) dye attached to a thermo labile azo linker (2,2'-azobis[ $N$-(2-carboxyethyl)-2methylpropionamidine]) is bound to the particle surface through PEG spacers of distinct molecular weight, and the 


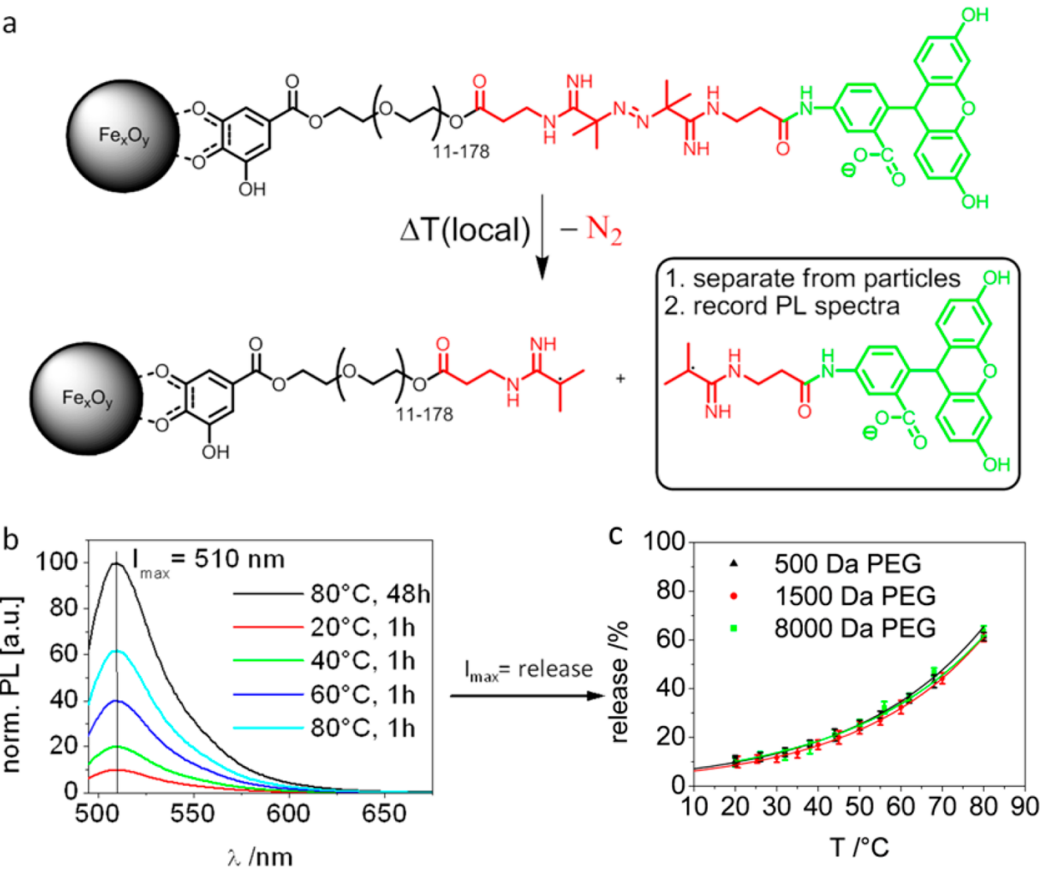

Figure 2. Calibration curves. (a) Sketch of the readily functionalized IONPs bearing FA connected through VA057 azo molecule to the tails of PEG spacers of different molecular weights. An increase in temperature results in increased cleavage of the azo group and release of the dye from the particles. The released FA is separated from the nanoparticles by centrifugation, and the PL spectra of the downstreams are recorded. (b) Calibration curves. Samples of IONP-PEG-azo-FA $(5 \mathrm{nM})$ were incubated for $1 \mathrm{~h}$ at temperature from 20 to $80{ }^{\circ} \mathrm{C}$; released FA was separated, and the PL spectra were recorded. Intensities were normalized to the maximum release (IONP-PEG-azo-FA sample incubated for $48 \mathrm{~h}$ at $80{ }^{\circ} \mathrm{C}$ ). (c) Quantitative correlations between the PL of the downstreams were obtained by plotting the release versus the incubation temperature and by fitting the data point with exponential functions. The error bars display the standard deviation.

dye release is monitored by photoluminescence. In this setup the IONPs are the heat mediators, the azo group is the thermometer, and the FA allows for the optical read-out of the absolute temperature, while the PEG linker provides the spacer, thus allowing subnanometer spatial resolution of the temperature measurement.

Finally, herein, the temperature stimulation concept was extended for demonstrating controlled drug delivery. FA was replaced with the chemotherapeutic agent doxorubicin (DOX) in the same nanoparticle arrangement; under actuation by AMF distance dependent control over the release rate of DOX was achieved and proven in vitro for $\mathrm{KB}$ cancer cells treatment.

We exploit the thermo labile molecule named azobis $[\mathrm{N}-(2$ carboxyethyl)-2-methylpropionamidine] containing the azo group to measure, upon excitation with AMFs $(f=334.5$ $\mathrm{kHz}, 9-17 \mathrm{mT})$, the local temperature increase of monodisperse superparamagnetic IONPs of $15 \mathrm{~nm}$ in diameter. Under these conditions, the contribution of eddy currents is negligible, $^{22}$ and only heating mechanisms like for instance hysteresis losses contribute to the local temperature increase. The magnetic characterization of PEGylated IONPs indicated a blocking temperature of $238 \mathrm{~K}$, a saturation magnetization of $58 \mathrm{emu} / \mathrm{g}$, and a strong exchange bias interaction of $275 \mathrm{Oe}$ together with a large coercive field of 2225 Oe (see Supporting Information (SI), Figures S5 and S6 and Table S6). FA bound to the azo molecule was used as detection unit for monitoring the thermal decomposition degree of the azo group covalently linked to the IONP surface, by measuring the photoluminescence spectra of the solution (see Figure 1). To vary the distance between the azo molecule and the nanoparticle surface, the azo molecules were attached to the nanoparticles through PEG spacers of different molecular weights. The amount of dye molecules released from the IONPs, at fixed time laps, is directly correlated to the decomposition degree of the azo group which itself depends on the (local) temperature $\left(T_{\text {local }}\right)$ at the IONP surface (for this specific azo molecule the $10 \mathrm{~h}$ half-life temperature in water is $57^{\circ} \mathrm{C}$ as stated by the supplier). Under AMF, the effective local heating can be expressed as the difference $\Delta T$ between the local temperature $T_{\text {local }}$ and the global temperature $T_{\text {global }}$ :

$$
\Delta T=T_{\text {local }}-T_{\text {global }}
$$

The global temperature is the temperature of the medium which surrounds the IONPs far away from their surface and can be measured with a temperature probe dipped into the solution.

For the IONP-PEG-azo-FA the local temperature can be obtained based on the decomposition rate of the azo group (see Figure 1). To obtain the quantitative correlation between released FA and the local temperature, calibration curves were recorded at different temperatures and fixed time laps. For these experiments, three stable colloidal solutions of IONPPEG-azo-FA $\left(M_{\mathrm{w}}(\mathrm{PEG})=500,1500\right.$, and $\left.8000 \mathrm{Da}\right)$ at a $5 \mathrm{nM}$ particle concentration in sodium borate buffer ( $\mathrm{pH} 9$, SBB9) were prepared and incubated for one hour at different temperatures, ranging from 20 to $80{ }^{\circ} \mathrm{C}$. Incubation in a water bath ensures the same temperature over the entire sample, being in this case the global temperature equal to the local temperature. The released dye was quickly separated from the IONPs by centrifugation on a membrane filter (molecular cutoff $=100 \mathrm{kDa}$ ), and the PL spectra of the downstreams were recorded. The PL signal $\left(I_{\text {max,incubation }}\right)$ increases with increasing temperature (see Figure $2 \mathrm{~b}$ ). To normalize the PL maxima at $510 \mathrm{~nm}$, the three samples at a $5 \mathrm{nM}$ particle concentration 
$\left(M_{\mathrm{w}}(\mathrm{PEG})=500,1500\right.$, and $\left.8000 \mathrm{Da}\right)$ were incubated at $80{ }^{\circ} \mathrm{C}$ for $48 \mathrm{~h}$, and the released dye (100\% release, see Supporting Information Figure S7 for more details) was separated from the IONPs by centrifugal filtration. The PL spectra of the downstreams were recorded, and $I_{\text {max,incubation was normalized }}$ to $I_{\max , 80^{\circ} \mathrm{C}, 48 \mathrm{~h}}$.

The normalized PL intensities $\left(I_{\max }=I_{\text {max, incubation }} / I_{\max , 80^{\circ} \mathrm{C}, 48 \mathrm{~h}}\right.$ $\times 100$ ), which represent the percentage of dye release, were plotted as a function of the incubation temperature and the data points fitted to an exponential function: $I_{\max }=A \mathrm{e}^{(T / \tau)}(T$ $=$ incubation temperature; $\tau=$ decay rate; see Figure $2 \mathrm{c}$ ). Comparable fitting parameters $A$ and $\tau$ for the three different samples were found (see Table S1). This indicates that the decomposition rate of the azo group is independent of the spacer molecule and its molecular weight (to break the azo bond always the same energy is required, and this does not depend on the molecular weight of the PEG). Having these calibration curves and by measuring the normalized PL intensity at $510 \mathrm{~nm}$ for samples exposed to an AMF, the local temperature at distinct distances from the nanoparticle surface can be extrapolated by using the following expression:

$$
T_{\text {local }}=\ln \left(\frac{I_{\max }}{A}\right) \times \tau
$$

Samples of IONP-PEG-azo-FA $\left(M_{\mathrm{w}}(\mathrm{PEG})=500,1500\right.$, and $8000 \mathrm{Da})$ at a $5 \mathrm{nM}$ particle concentration were placed in a hyperthermia device (magneTherm AC system Nanotherics Corp.). The solution temperature $\left(T_{\text {global }}\right)$ was probed by placing an optical fiber into the sample solution, and the temperature stayed constant over the entire measurement $( \pm 1$ ${ }^{\circ} \mathrm{C}$ for $1 \mathrm{~h}$ ). The magnetic field amplitude $B$ can be tuned by changing the applied voltage on the coil. Magnetic field amplitudes spanning 9-17 mT $\left(7-13.5 \mathrm{kAm}^{-1}\right)$ at $334.5 \mathrm{kHz}$ were applied for $1 \mathrm{~h}$, and the released dye was quickly separated from the particles by centrifugation in a centrifuge filter (MWCO $100 \mathrm{kDa}$ ). The PL spectra of the downstreams were recorded, and the resulting $I_{\mathrm{max}, \mathrm{AMF}}$ were normalized $\left(I_{\mathrm{AFM}}=\right.$ $\left.I_{\max , \mathrm{AFM}} / I_{\max , 80^{\circ} \mathrm{C}, 48 \mathrm{~h}} \times 100\right)$. With eqs 1 and 2 and knowing the $T_{\text {global }}$, the difference between the local and the global temperature $\Delta T$ was calculated. In Figure $3 a$ the $\Delta T$ values as a function of the applied magnetic field amplitudes are reported for the three samples at different molecular weights of PEG. Significant $\Delta T$ values up to $45 \mathrm{~K}$ can be reached if the azo-FA group is linked through a very short spacer (500 Da PEG) to the IONP and the highest magnetic field amplitude $(17 \mathrm{mT})$ is applied (see Table S2). On the contrary, longer PEG spacers lead to $\Delta T$ values around $10 \mathrm{~K}$ for the same magnetic field amplitude. Two clear trends can be observed: $\Delta T$ linearly increases with increasing magnetic field amplitude $B$ and decreases exponentially with the increasing molecular weight of the PEG spacer. This leads to the conclusion that $\Delta T$ is a function of the applied magnetic field amplitude (at a given frequency) and of the molecular weight of the PEG spacer (while the latter one represents the predominant effect due to exponential rather than a linear correlation between $\Delta T$ and distance).

Different control tests were performed. First, in a control experiment we exposed ultrasmall IONPs (6 nm in diameter) bearing Gallol-1500 kDaPEG-azo-FA and repeated the AMF experiments (see SI, Figure S8). These particles have been shown to develop no local temperature increase. ${ }^{22}$ Indeed we obtained $\Delta T$ values below $5 \mathrm{~K}$ for these ultrasmall IONPs. This
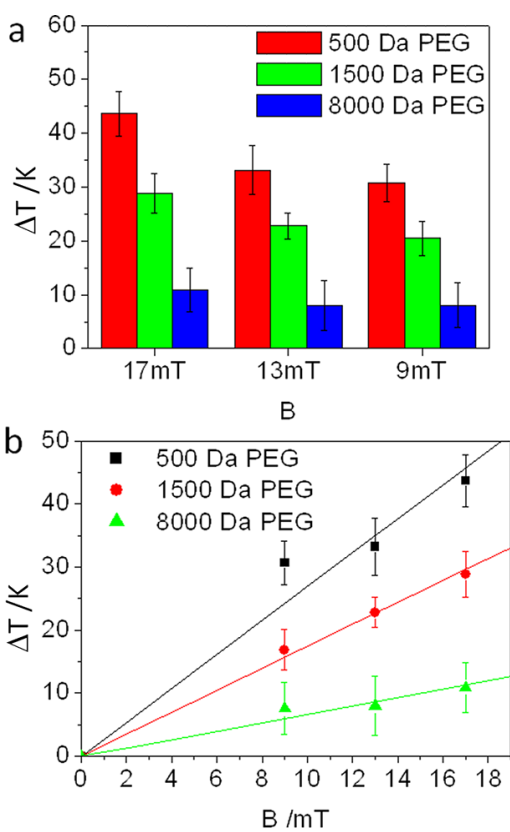

Figure 3. Measured temperature profiles. (a) $\Delta T$ values as a function of applied magnetic field amplitudes and $M_{\mathrm{w}}(\mathrm{PEG})$ after $1 \mathrm{~h}$ of AMF treatment are plotted. $\Delta T$ decreases with decreasing field amplitudes and increasing $M_{\mathrm{w}}$ (PEG). (b) $\Delta T$ plotted as a function of field amplitude reveals linearly increasing values for all $M_{\mathrm{w}}(\mathrm{PEG})$.

experiment clearly proves two points: (i) the local temperature change and not the magnetic field itself induces the thermal decomposition of the azo linker; (ii) the intrinsic properties of the IONP are crucial for efficient local heat generation. Additional control tests were performed to ensure that the FA release was due exclusively to the decomposition of the azo group, while the PEG molecules remained attached at the nanoparticle surface during the whole heat treatment. To this aim, a sample of IONP-PEG-azo-FA $\left(M_{\mathrm{w}}(\mathrm{PEG})=1.5 \mathrm{kDa}\right)$ was heated for $24 \mathrm{~h}$ at $80{ }^{\circ} \mathrm{C}$, and by centrifugation the nanoparticles (collected on the upper part of the Amicon filters, MWCO $100 \mathrm{kDa}$ ) were separated from the downstream (eventually the downstream would contain any PEG derivate molecules which have come off from the nanoparticle surface). The latter was analyzed by UV-vis spectroscopy: no signal corresponding to PEG molecules was detected, while the FTIR spectra of the separated IONPs, collected on the filter, exhibited the characteristic PEG signals (see Figure S9 of the Supporting Information). Additionally, to verify that the azo molecule is covalently attached to the PEG, ${ }^{1} \mathrm{H}$-NMR was used, and to this aim, first the PEG ligands were stripped off from the nanoparticles (NMR cannot be performed directly on the magnetic nanoparticles): this was achieved by incubating an aliquot of IONPs@GA-PEG1500-azo sample in acidified brine ( $\mathrm{pH} 1$ ) for 2 days. Soon after, the phase extraction of GA-PEG/ GA-PEG-azo from the aqueous phase to the ice cold chloroform allows to separate ligands from the nanoparticles and to recover only the latter in the chloroform phase. ${ }^{1} \mathrm{H}$ NMR analysis on this phase showed a small shift for the signals derived from the azo molecule bound to PEG (in respect to free azo, VA057). Moreover, since the azo molecule itself is insoluble in chloroform, the presence of the signal from VA057 in the chloroform phase also indicates the linkage to the PEG (see SI, Figure S10). Covalent linkage of FA to the azo end group was additionally assessed by high-performance liquid 

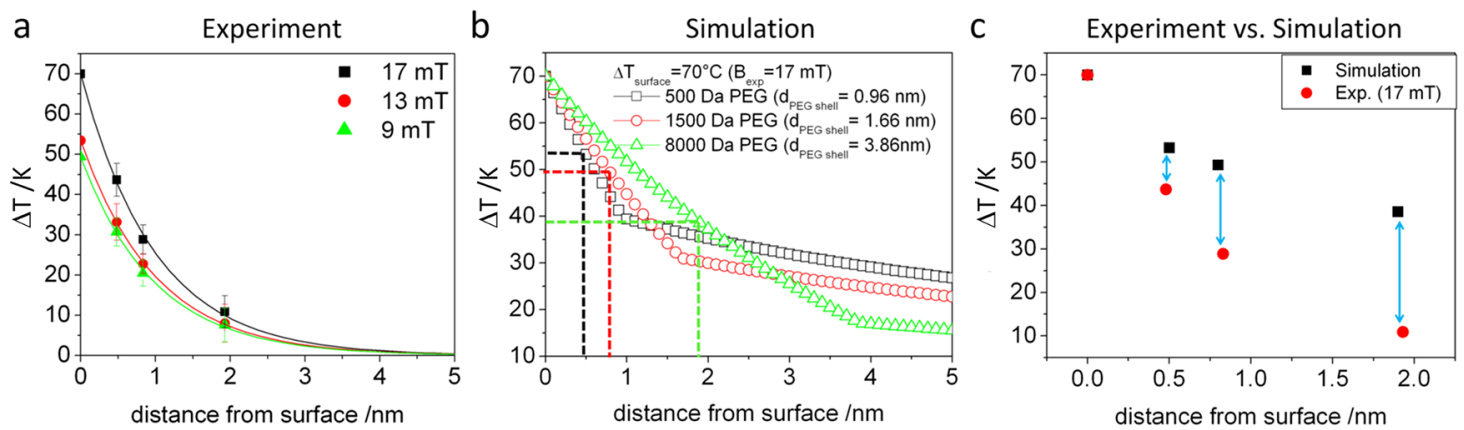

Figure 4. (a) Experimental temperature gradients for all field amplitudes: significant local-to-global temperature differences can be found at distances shorter than $3 \mathrm{~nm}$. (b) Temperature gradients calculated by applying the Fourier law for three different shell thicknesses (PEG500, PEG1500, and PEG8000). (c) Comparison of $\Delta T$ values at $0,0.47,0.83$, and $1.9 \mathrm{~nm}$. The discrepancy between experimental data and the diffusive heat transport model at this nanoscale regime can be observed and is increasing with distance.

chromatography (HPLC) analysis. Unmodified FA exhibited a significantly different retention time than FA released from the nanoparticles after incubation (see SI, Figure S11).

It is worthy of notice that the hydrolysis of the ester bonds in the molecular probe would represent a limitation to the use of our system as thermo-probe. To verify that no hydrolysis of the ester bonds occurred in our experimental conditions, an additional control experiment was performed. A concentrated sample of IONP-GA-PEG1500 (13 $\mathrm{mg}$ of $\mathrm{Fe}$ in $3 \mathrm{~mL})$ in sodium borate buffer $(\mathrm{pH} 9)$ was incubated $48 \mathrm{~h}$ at $80^{\circ} \mathrm{C}$ (this time scale is much longer than the $1 \mathrm{~h}$ hyperthermia treatment usually applied in the experiments described above), and the downstream after centrifugal filtration was collected. The presence of PEG molecules in the downstream would indicate the hydrolysis of the ester bond. By thermogravimetric analysis we could assess that the amount of PEG at the nanoparticle surface corresponds to about $10 \%$ in weight (see Figure S12). Knowing that PEG has a characteristic UV absorption (with a strong absorption at $200 \mathrm{~nm}$ and a peak around $240 \mathrm{~nm}$ ), by comparing the absorption spectra of a PEG solution $(1.3 \mathrm{mg}$ in $3 \mathrm{~mL}$ SBB9 about $10 \%$ in weight of the nanoparticle solution) to that of the downstream, it can be concluded that the amount of PEG released from the nanoparticles is negligible (Figure S13). Overall, by these tests we could assess that the FA release is dependent predominantly on the azo decomposition under AFM treatment of the nanoparticles.

In Figure $3 \mathrm{~b}, \Delta T$ is plotted versus $B$, showing for all PEG spacers a linear dependence with $B$. Depending on the molecular weight, the slope of the linear fit varies (see also Table S3a): higher molecular weights result in lower slopes, which can be explained by the different distance of the azo-FA end group to the excited IONP: The closer the thermo labile group is to the infinite cooling source (the aqueous solution) and thus the farther is the azo group from the nanoparticle surface, the less significant is the increase in local temperature with increasing fields. It should be pointed out that one expects that $\Delta T$ follows a power law with $B$ as the SAR does for superparamagnetic nanocrystals. ${ }^{23}$ This deviation may be attributed to the assumption of adiabatic conditions during a typical SAR determination experiment, which are far from the conditions used in our experiments. In diluted conditions and for excitation over long periods the system reaches an equilibrium state where both the global and the local temperature stay constant over time. The medium in which the IONPs are dispersed serves as an infinite cooling source, and the heat flux from the IONP surface stays constant. We can conclude from our experiments that, under isothermal, equilibrium conditions, the increase in temperature at the IONPs surface scales linearly with the applied magnetic field.

To obtain the field-dependent heat gradients, the molecular weight of PEG can be used to calculate the average distance of the azo-FA end group to the surface. Previous FCS experiments have shown that for low molecular weight PEG bound to nanoparticles, the best fit of the radius of gyration $R_{\mathrm{G}}$ in a random polymeric coil is given by the following expression: ${ }^{24}$

$$
R_{\mathrm{G}}=l \cdot \sqrt{\frac{M_{\mathrm{w}}}{6 \cdot M_{\text {monomer }}}}
$$

where $l$ is the length of one monomer unit, $M_{\mathrm{w}}$ is the molecular weight of the polymer, and $M_{\text {monomer }}$ is the molecular weight of the monomer. $R_{\mathrm{G}}$ is proportional to the square root of $M_{\mathrm{w}}$, which means that low molecular weight polymers are present in a more stretched conformation than higher molecular weight polymers. In the case of PEG, $l$ is equal to $0.35 \mathrm{~nm}, M_{\text {monomer }}$ is $44.05 \mathrm{~g} \cdot \mathrm{mol}^{-1}$, and the resulting $R_{\mathrm{G}}$ values are summarized in Table S4. In a random coil the end group is located on average in the middle of the coil, which means that the azo-FA end group is at a distance from the IONP surface equal to $R_{\mathrm{G}}{ }^{24}$

In Figure $4 a, \Delta T$ is plotted versus the distance to the surface of the azo-FA end group $\left(R_{\mathrm{G}}\right)$, showing steep exponential decays of $\Delta T$ with increasing distance from the surface for all applied magnetic field amplitudes. For these IONPs, significant local temperature differences can be found in the range from 0 to $3 \mathrm{~nm}$. The data points are numerically fitted with exponential decay functions of the type $\Delta T=A \times \mathrm{e}^{-(d / \tau)}$, where $d$ is the distance of the end group to the surface (see SI, Table S3b for fitting parameters). The difference between local and global temperature at the surface $(d=0 \mathrm{~nm})$ can be obtained by extrapolation of the fitting functions to $d \rightarrow O$ resulting in $\Delta T=$ $A$. At the surface, the absolute temperature is $T_{\mathrm{abs}}=\Delta T_{d=0}+$ $T_{\text {global }}$ (see SI, Table S5). At the highest field amplitude applied $(17 \mathrm{mT})$, absolute temperatures around the boiling point of water were reached. Additionally, these data also demonstrate that under AMF and in diluted conditions the heat generated at the IONP surface is quite significant; however it is limited to the immediate proximity of the nanoparticle surface.

Calculations based on the Fourier law using the thermal conductivity of polymers and water predict that the temperature profile should decay slower as we move apart from the NP surface than the experimental results observed by us (see Figure $4 b$ and c). The reason for such a discrepancy is that we are measuring the temperature profile in a nanometric region 
a
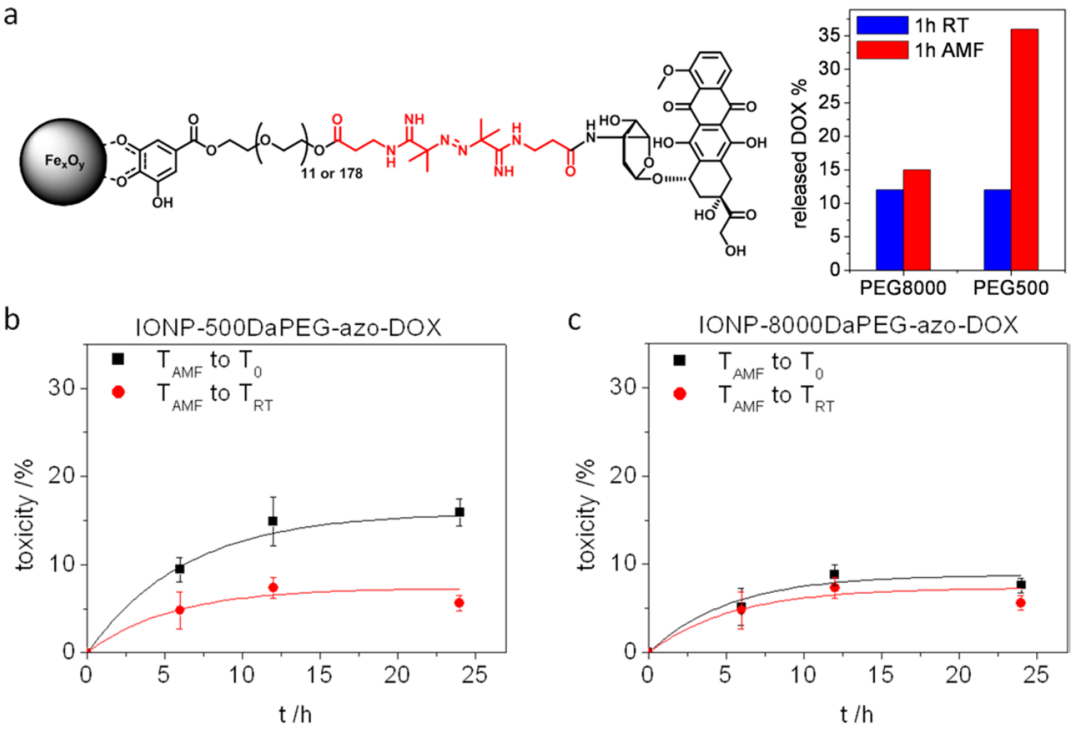

b
C

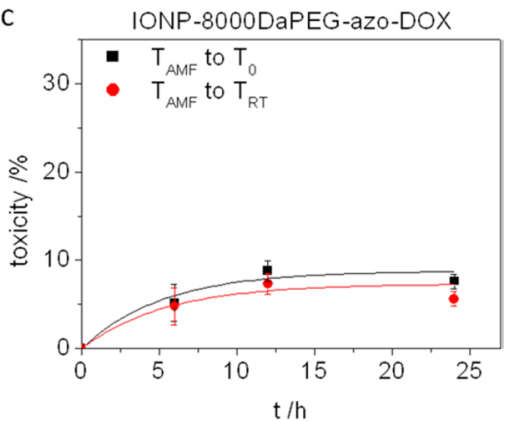

Figure 5. Toxicity experiments. (a) Sketch of the readily functionalized IONPs bearing DOX which is covalently linked through VA057 azo to the PEG spacers of different two different $M_{\mathrm{w}}(500$ and $8000 \mathrm{Da})$ together with the release profiles for samples exposed for $1 \mathrm{~h}$ at RT and under AMF $(334.5 \mathrm{kHz}, 17 \mathrm{mT})$, respectively. (b) Cytotoxicity within $24 \mathrm{~h}$ of DOX released from IONPs-500 Da PEG after AFM treatment compared to a sample incubated $1 \mathrm{~h}$ at RT $\left(T_{\mathrm{RT}}\right)$ and to a blank kept for $1 \mathrm{~h}$ at RT $\left(T_{0}\right)$. (c) Cytotoxicity of DOX released from IONPs-8000 Da PEG after AFM treatment compared to a sample incubated $1 \mathrm{~h}$ at RT $\left(T_{\mathrm{RT}}\right)$ and to a blank $\left(T_{0}\right)$.

with dimension similar (or even smaller) to that of the phonons' mean free path. In these conditions, the heat transport is not diffusive but ballistic, and the Fourier law is not valid anymore. ${ }^{25}$ Calculations carried out by us considering these nanoscale thermal effects are in agreement with the experimental observations (see SI, Figure S15).

The fast temperature decay profile was supported also by recently reported results in which ion channels in neurons or plasma glucose can be remotely regulated by magnetic nanoparticles exposure to AMF. ${ }^{14,26}$ In analogy to our study, also in those studies the macroscopic temperature was kept well below the temperature needed to activate a specific biological effect, that is, the thermal activation of the ion channel. This suggests that the effects were due to higher temperature reached only locally at the cell membrane. We found also that the local heating effects are not significantly influenced by the concentration of the NPs within a defined range (between 2 and $50 \mathrm{nM}$ ). Indeed we found the same release percentage, and hence $\Delta T$ values, when we used 10 times more concentrated nanoparticle solutions (data not shown). At concentration below $2 \mathrm{nM}$ the detection limit of the fluorophore FA is reached. These results are clearly significant for the design and application of AMF triggered drug release systems.

Our findings can be indeed directly implemented in an AMF triggered drug release system if the fluorescent dye FA is replaced by doxorubicine (DOX). Control over the DOX release rate could be achieved by positioning the thermo labile group at distinct distances from the IONPs, which results in different maximum $\Delta T$ values when the AMF is applied. To this end we synthesized IONP-PEG-azo-DOX compounds using PEG spacers of two different molecular weights (500 and 8000 Da, see Figure 5a).

The two IONP samples at distinct PEG molecular weight were dispersed in cold cell culture medium, and each of them was split in three aliquots. The first aliquot was centrifuged directly after the preparation in order to precipitate the IONPs, and the supernatants were collected and served as blanks $\left(T_{0}\right.$, no DOX release should be expected). One aliquot was incubated at room temperature $\left(T_{\mathrm{RT}}\right)$ for one hour and never exposed to AMF before centrifugation and collection of the supernatant. These samples provided the control for the release due to the thermal decomposition of the azo at room temperature (RT) (the temperature of the medium was equal to RT during the entire AFM treatment). For both IONP samples coated with PEG of distinct molecular weights, about $12 \%$ of DOX was released at RT, which can be ascribed to the thermal decomposition of the azo group. The third aliquot of each sample was treated for $1 \mathrm{~h}$ at $334.5 \mathrm{kHz}$ and $17 \mathrm{mT}\left(T_{\text {hyp }}\right)$. The amount of DOX released via hyperthermia does depend on the local temperature on the IONPs surface during the AMF treatment: for the shorter PEG (500 Da), about $36 \%$ of DOX was released from the IONPs, while for the compound bearing the longer PEG spacers (8000 Da) only $15 \%$ of DOX was released (see Figure 5a). These results are in good agreement with the experiments with FA and lead to the conclusion that neither the suspension media nor the IONP concentration or the different molecules (DOX instead of FA) bound to the azo end group affect the decomposition rate of the azo group.

To cross check the release capability of our system, in vitro experiments were carried out, and the release was monitored by studying the cytotoxicity of the DOX on tumor cells. In this regard, for cell toxicity experiments, supernatants $\left(T_{0}, T_{\mathrm{RT}}\right.$, and $T_{\text {hyp }}$ containing different amount of DOX released from the nanoparticles) were used to treat $\mathrm{KB}$ cells for 6,12 , and $24 \mathrm{~h}$, and the cytotoxicity effects was measured with a viability assay (PrestoBlue Reagent, Invitrogen). The fluorescence signals at $590 \mathrm{~nm}$ recorded before (as blank) and after the addition of the PrestoBlue reagent allowed us to read the number of living cells for each sample. Upon AMF treatment, the IONP-500 DaPEGAzo-DOX released a significant amount of DOX with respect to control samples at $T_{0}$ and at $T_{\mathrm{RT}}$ (see Figure $5 \mathrm{~b}$ and c), while the 8000 Da PEG conjugate did not. Hence, the cytotoxicity of the sample with the short spacer was three times higher than that of the sample bearing the longer spacer. This clearly demonstrates the remarkable distance dependence of thermally 
induced decomposition of the azo group, the linker between the drug and the PEGylated IONP.

A further proof of the heat generated by the IONPs under AMF came by comparing the magnetic features of the nanoparticles before and after exposure to AMF or to a heat annealing process (see SI, Figures S16 and S17). There were no differences in magnetic properties between PEG coated NPs at different molecular weights (see SI, Figure S6 and Table S6). On the other hand, remarkable improvements of the magnetic properties (i.e., lower coercive fields and higher saturation magnetization) were observed for those samples exposed either to a AMF or to an annealing process with respect to the asprepared nanocrystals (see SI, Figures S16 and S17 and Table S6). X-ray diffraction (XRD) analysis of a sample before and after annealing at $140{ }^{\circ} \mathrm{C}$ for $2 \mathrm{~h}$ revealed the reason for the change in magnetic properties. Before annealing the IONPs were mainly composed of $\mathrm{Fe}_{3} \mathrm{O}_{4}$ but contained also some metallic Fe. After annealing, the Fe peaks disappeared, and only peaks ascribable to $\mathrm{Fe}_{3} \mathrm{O}_{4}$ were present (see SI, Figure S18). Taking into account the improvement of both annealed and AFM exposed nanoparticles, it can be concluded that for the latter a similar annealing process takes place, and since no direct heat is applied here, only a local heating effect can lead to the evolution of the magnetic features. These data demonstrate indirectly the local heat at the nanoparticle surface under AMF.

In conclusion, in this work we have demonstrated that thermo-labile azo-ligands anchored to superparamagnetic nanoparticles via PEG spacers can be utilized for: (i) spatially resolved measurement of temperature surface profile on dilute solutions of iron oxide nanoparticles exposed to an AMF and (ii) as linker between the cytostatic drug doxorubicin and a PEGylated iron oxide nanoparticle for AMF triggered drug release. By varying the molecular weight of the PEG spacer, the distance between the IONPs surface and the azo group could be tuned, and thus the local temperature as a function of the distance to the nanoparticle surface could be assessed. Upon excitation with an AMF, the local nanoparticle temperature decays rapidly with increasing distance. Furthermore, we found that $\Delta T$ scales linearly with the applied fields for all molecular weights of the PEG under isothermal conditions. Also, in the drug release experiment, this carrier shows the following promising features as smart drug delivery systems: (i) the mass ratio between the delivery vehicle and the drug is significantly reduced; (ii) hydrophobic as well as hydrophilic drugs can be bound; (iii) the drug release rate can be adjusted based on molecular building blocks (i.e., the azo molecules); and (iv) combinations of different drugs bound on the same nanoparticle at different distances would enable independent release of each of them. The work here presented not only gives access to a new type of temperature sensors at the nanoscale for measuring the surface temperature gradient around magnetic nanocrystals under AMF but will allow for remote triggering of combinatorial drug delivery. Eventually, more than one drug will be released with different kinetics and without any macroscopic heating, thus avoiding collateral effects to the surrounding tissues in proximity of tumors. Finally, the local heating was here indirectly observed by means of the improvement of the magnetic parameters of the initial nanoparticle. These nanoparticles indeed undergo a structural transformation while being exposed to an alternating magnetic field. This last result might disclose a new type of hyperthermia treatment as the heating performance of the particles can be improved during the treatment.

\section{ASSOCIATED CONTENT}

\section{S Supporting Information}

Experimental sections, additional ZFC, FC magnetic measurements, XRD data, control experiments including TEM images, ${ }^{1} \mathrm{H}$ NMR, UV-vis and PL spectra, HPLC analysis, and mathematical modeling. This material is available free of charge via the Internet at http://pubs.acs.org.

\section{AUTHOR INFORMATION}

\section{Corresponding Author}

*E-mail: teresa.pellegrino@iit.it.

Notes

The authors declare no competing financial interest.

\section{ACKNOWLEDGMENTS}

This work was supported in part by the European project Magnifyco (ContractNMP4-SL-2009-228622) and by the Italian FIRB grant "Ossidi Nanostrutturati” (contract \#RBAP115AYN). We thank Simone Nitti for providing Iron Oxide NPs and Giammarino Pugliese for his technical support and Mauro Povia for assistance with the XRD measurements.

\section{REFERENCES}

(1) Kong, G.; W. D., M. Review Hyperthermia and liposomes. Int. J. Hypertherm. 1999, 15 (5), 345-370.

(2) Kumar, C. S. S. R.; Mohammad, F. Magnetic nanomaterials for hyperthermia-based therapy and controlled drug delivery. Adv. Drug Delivery Rev. 2011, 63 (9), 789-808.

(3) Guardia, P.; Di Corato, R.; Lartigue, L.; Wilhelm, C.; Espinosa, A.; Garcia-Hernandez, M.; Gazeau, F.; Manna, L.; Pellegrino, T. Water-Soluble Iron Oxide Nanocubes with High Values of Specific Absorption Rate for Cancer Cell Hyperthermia Treatment. ACS Nano 2012, 6 (4), 3080-3091.

(4) Lee, J.-H.; Jang, J.-t.; Choi, J.-s.; Moon, S. H.; Noh, S.-h.; Kim, J.w.; Kim, J.-G.; Kim, I.-S.; Park, K. I.; Cheon, J. Exchange-coupled magnetic nanoparticles for efficient heat induction. Nat. Nanotechnol. 2011, 6 (7), 418-422.

(5) Laurent, S.; Dutz, S.; HÃafeli, U. O.; Mahmoudi, M. Magnetic fluid hyperthermia: Focus on superparamagnetic iron oxide nanoparticles. Adv. Colloid Interface Sci. 2011, 166 (1-2), 8-23.

(6) Gazeau, F.; Levy, M.; Wilhelm, C. Optimizing magnetic nanoparticle design for nanothermotherapy. Nanomedicine 2008, 3 (6), 831-844.

(7) Bae, K. H.; Park, M.; Do, M. J.; Lee, N.; Ryu, J. H.; Kim, G. W.; Kim, C.; Park, T. G.; Hyeon, T. Chitosan Oligosaccharide-Stabilized Ferrimagnetic Iron Oxide Nanocubes for Magnetically Modulated Cancer Hyperthermia. ACS Nano 2012, 6 (6), 5266-5273.

(8) Mornet, S.; Vasseur, S.; Grasset, F.; Duguet, E. Magnetic nanoparticle design for medical diagnosis and therapy. J. Mater. Chem. 2004, 14 (14), 2161-2175.

(9) Bothun, G. D.; Preiss, M. R. Bilayer heating in magnetite nanoparticle liposome dispersions via fluorescence anisotropy. $J$. Colloid Interface Sci. 2011, 357 (1), 70-74.

(10) Ruiz-Hernández, E.; Baeza, A.; Vallet-Regí, M. A. Smart Drug Delivery through DNA/Magnetic Nanoparticle Gates. ACS Nano 2011, 5 (2), 1259-1266.

(11) Dobson, J. Remote control of cellular behaviour with magnetic nanoparticles. Nat. Nanotechnol. 2008, 3 (3), 139-143.

(12) Keblinski, P.; Cahill, D. G.; Bodapati, A.; Sullivan, C. R.; Taton, T. A. Limits of localized heating by electromagnetically excited nanoparticles. J. Appl. Phys. 2006, 100 (5), 054305.

(13) Creixell, M.; Bohórquez, A. C.; Torres-Lugo, M.; Rinaldi, C. EGFR-Targeted Magnetic Nanoparticle Heaters Kill Cancer Cells without a Perceptible Temperature Rise. ACS Nano 2011, 5 (9), $7124-7129$. 
(14) Huang, H.; Delikanli, S.; Zeng, H.; Ferkey, D. M.; Pralle, A. Remote control of ion channels and neurons through magnetic-field heating of nanoparticles. Nat. Nanotechnol. 2010, 5 (8), 602-606.

(15) Okabe, K.; Inada, N.; Gota, C.; Harada, Y.; Funatsu, T.; Uchiyama, S. Intracellular temperature mapping with a fluorescent polymeric thermometer and fluorescence lifetime imaging microscopy. Nat. Commun. 2012, 3 (705), 1-9.

(16) Yang, J.-M.; Yang, H.; Lin, L. Quantum Dot Nano Thermometers Reveal Heterogeneous Local Thermogenesis in Living Cells. ACS Nano 2011, 5 (6), 5067-5071.

(17) Hamad-Schifferli, K.; Schwartz, J. J.; Santos, A. T.; Zhang, S. G.; Jacobson, J. M. Remote electronic control of DNA hybridization through inductive coupling to an attached metal nanocrystal antenna. Nature 2002, 415 (6868), 152-155.

(18) Orfeuil, M. Electric Process Heating: Technologies/Equipment/ Applications; Battelle Press: Columbus, OH, 1987.

(19) Lee, J.; Govorov, A. O.; Kotov, N. A. Nanoparticle Assemblies with Molecular Springs: A Nanoscale Thermometer. Angew. Chem. 2005, 117 (45), 7605-7608.

(20) Brites, C. D. S.; Lima, P. P.; Silva, N. J. O.; Millán, A.; Amaral, V. S.; Palacio, F.; Carlos, L. D., A Luminescent Molecular Thermometer for Long-Term Absolute Temperature Measurements at the Nanoscale. Adv. Mater. 22, (40), 4499-4504.

(21) Polo-Corrales, L.; Rinaldi, C. Monitoring iron oxide nanoparticle surface temperature in an alternating magnetic field using thermoresponsive fluorescent polymers. J. Appl. Phys. 2012, 111 (7), 07B334.

(22) Gupta, A.; Kane, R. S.; Borca-Tasciuc, D. A., Local temperature measurement in the vicinity of electromagnetically heated magnetite and gold nanoparticles. J. Appl. Phys. 2010, 108, (6), art. no. 064901.

(23) Rosensweig, R. E. Heating magnetic fluid with alternating magnetic field. J. Magn. Magn. Mater. 2002, 252 (1-3 SPEC. ISS.), $370-374$.

(24) Riedinger, A.; Zhang, F.; Dommershausen, F.; Röcker, C.; Brandholt, S.; Nienhaus, G. U.; Koert, U.; Parak, W. J. Ratiometric Optical Sensing of Chloride Ions with Organic Fluorophore-Gold Nanoparticle Hybrids: A Systematic Study of Design Parameters and Surface Charge Effects. Small 2010, 6 (22), 2590-2597.

(25) Chen, G. Nonlocal and Nonequilibrium Heat Conduction in the Vicinity of Nanoparticles. J. Heat Transfer 1996, 118 (3), 539-545.

(26) Stanley, S. A.; Gagner, J. E.; Damanpour, S.; Yoshida, M.; Dordick, J. S.; Friedman, J. M. Radio-Wave Heating of Iron Oxide Nanoparticles Can Regulate Plasma Glucose in Mice. Science 2012, 336 (6081), 604-608. 